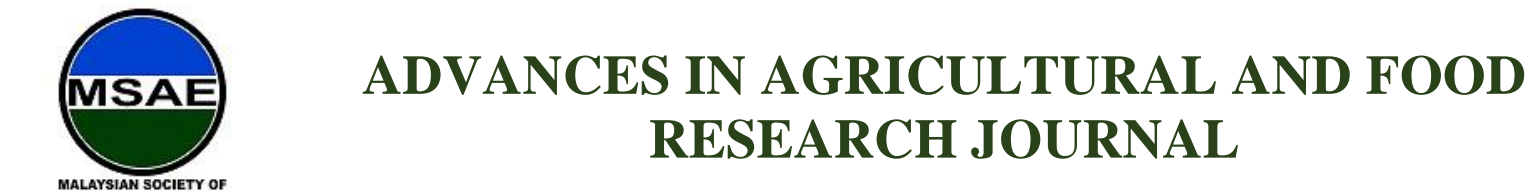

Review Article

\title{
Towards Cultivating Black Pepper Using Geospatial Technology for Growth Monitoring and Mapping: A Review for Small Scale Practice in Malaysia
}

Siti Zul Lailee Kamsan ${ }^{1 *}$, Wan Nor Zanariah Zainol@ Abdullah ${ }^{2}$, Rosnah Shamsudin ${ }^{3}$

${ }^{1}$ Department of Basic Sciences and Engineering, Universiti Putra Malaysia Bintulu Sarawak Campus, Bintulu, 97008, Malaysia, zullaileekamsan@gmail.com

${ }^{2}$ Department of Basic Sciences and Engineering, Universiti Putra Malaysia Bintulu Sarawak Campus, Bintulu, 97008, Malaysia,wnzz@upm.edu.my

${ }^{3}$ Department of Process and Food Engineering, Faculty of Engineering, Universiti Putra Malaysia, 43400, Serdang, Selangor, Malaysia, rosnahs@upm.edu.my

*Corresponding author: Siti Zul Lailee Kamsan, Department of Basic Sciences and Engineering, Universiti Putra Malaysia Bintulu Sarawak Campus, Bintulu, 97008, Malaysia; zullaileekamsan@gmail.com

Abstract: Alternative to black pepper cultivation is crucial to level up the productivity of crops. Application of geospatial technology in black pepper cultivation which has been classified as small scale agricultural activity is seen to enhance their production. In this paper, we use a comprehensive study on journal papers, conference proceedings, formal web sites and books as fundamental sources of information. Firstly, the study reviews on current black pepper cultivation and related producers in Malaysia. Secondly, it focused on geospatial technology especially on remote sensing and crop growth monitoring that have been implemented and well established. Lastly, limitation to implement geospatial technology on black pepper cultivation in Malaysia situation was discussed. Therefore, it is predicted that geospatial technology will improve black pepper productivity and implementation of this technology on small scale cultivation requires consolidation from various agencies since it requires high investment and foundation.

Keywords: black pepper; precision farming; crop growth monitoring; crop mapping; remote sensing

Received: $14^{\text {th }}$ September 2020

Received in Revised Form: $19^{\text {th }}$ December 2020

Accepted: $24^{\text {th }}$ December 2020

Available Online: $11^{\text {th }}$ January 2021

Citation: Kamsan, S. Z. L., Zainol Abdullah, W. N. Z \& Shamsudin, R. Towards cultivating black pepper using geospatial technology for growth monitoring and mapping: A review for small scale practice in Malaysia. Adv Agri Food Res J 2021; $2(1)$ : a0000125. https://doi.org/10.36877/aafrj. a0000125

\section{Introduction}

Black pepper is one of the important global commodity crops with increasing demand due to its multipurpose usage in human life (Abraham, 2018). It was also known as the $3^{\text {rd }}$ 
most popular spices used in food ingredient (Kumar \& Swarupa, 2017). In recent, black pepper is grown in about 26 countries and occupies 500,000 ha with the productivity of 790.2 kg/ha (Krishnamoorthy \& Parthasarathy, 2009; Kumar et al., 2017; Paduit et al., 2018). Vietnam, Indonesia, Brazil, Malaysia, and Thailand were recorded among the highest production of pepper around the world (Teuscher et al., 2016; Jasni et al., 2017; Sanny et al., 2018). Among the producer countries of black pepper, Malaysia contributes 7-8\% of global market and it internationally traded as Sarawak pepper (Ravindran \& Kallupurackal, 2012). However, black pepper is categorized as one of the perennial crops with high demand of nutrients (Yap, 2012a; Sulok et al., 2018), low productivity due to management and pest disease problems (Rosli et al., 2013; Kamarudin et al., 2013; Yap \& Jarroop, 2018). Therefore, maintaining black pepper productivity is important to fulfil the demand for pepper market.

The National Policy on Industry 4.0 also known as Industry 4WRD is one of the initiatives by the government of Malaysia to enhance the implementation of precision agriculture in the industry (Ministry of International Trade and Industry, 2018). Precision agriculture has been popularized since the evolution of industrial revolution (IR) was introduced in the late 1960s. The concept of precision agriculture relies on monitoring, collecting and interpreting data which enable to create growing condition that is linked together continuously (Charyulu et al., 2019; Zambon et al., 2019). Besides, the concept requires producers and farmers to communicate with technology rather than operating them. This process integrates the combination of information technology tools to emphasize understanding on cultivated land and improve the management, quality of produces and environment respectively (Fardusi et al., 2017). Therefore, implementation of geospatial technology for black pepper cultivation is suggested to ensure the productivity of black pepper, healthy environment and profitable income in the future.

Hence, this paper has the following objectives (1) to identify the gap and requirement needed for black pepper cultivation to improve their productivity (2) to provide an overview on related geospatial technology suitable for black pepper growth monitoring and (3) to discuss the limitation of geospatial technology for small scale cultivation practices.

\section{Black Pepper Cultivation Scenario in Malaysia}

Black pepper is a perennial crop of humid tropics, requires an adequate amount of humidity, rainfall and temperature (Sivaraman et al., 1999; Srinivasan et al., 2007). Cultivation of black pepper is recommended at soils ranging from heavy soil to light sandy clays that rich in humus since nutrient absorption commonly focus on top $50-60 \mathrm{~cm}$ layer of the soil (Sivaraman et al., 1999). In Malaysia, black pepper mostly cultivated in Sarawak with laterites type of soil (Oxisol). However, the inadequacy of soil nutrient is one of the crucial problems notably for supplying nutrient to the crops (Abd Hamid \& Wan Yahaya, 2019). 
Black pepper are recommended to be planted at $2.0 \mathrm{~m} \times 2.4 \mathrm{~m}$ or $2.4 \mathrm{~m} \times 2.4 \mathrm{~m}$. Planting of cover crop and terracing are also suggested considering the hilly planting area with more than 40 degrees in most farm to conserve the soil (Malaysia Pepper Board, 2018b). Nonetheless, most of the farms in Sarawak, practising weeds cleaning with minimal consideration on the soil (Abd Hamid \& Wan Yahaya, 2019) and nutrient requirement for the crop growth. Concurrently, this commodity crop experience declining in market prices (see Figures 1 and 2) as a result of domestic and international market factors.

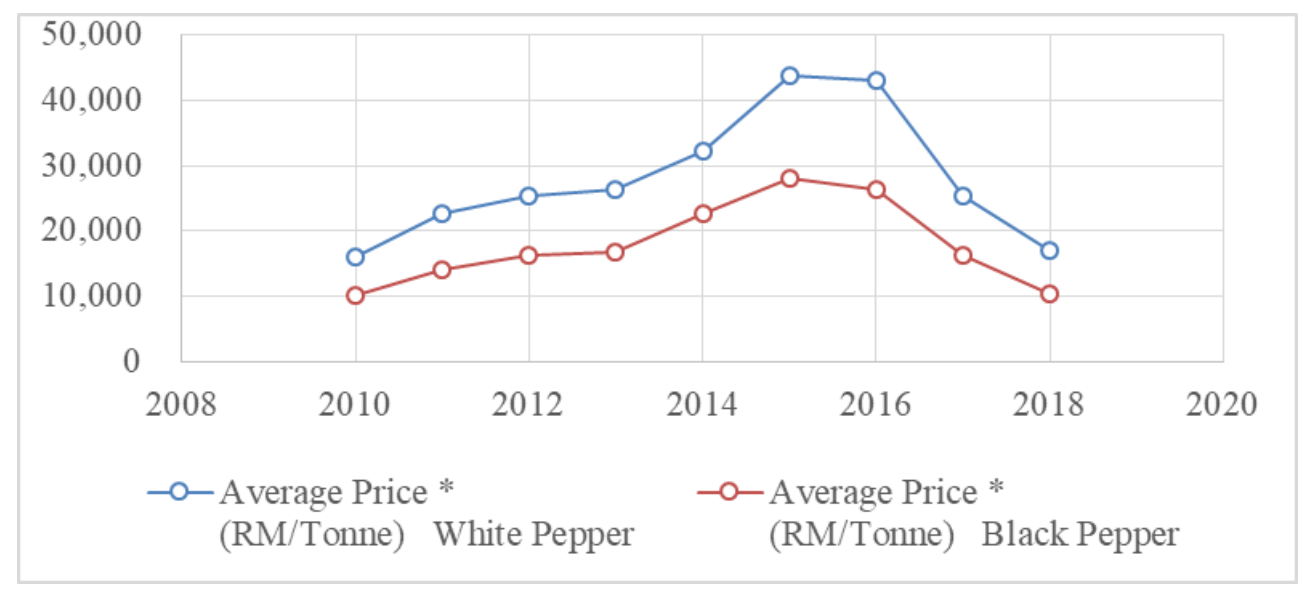

Figure 1. Average pepper price in Kuching, Sarawak (Malaysia Pepper Board, 2018).

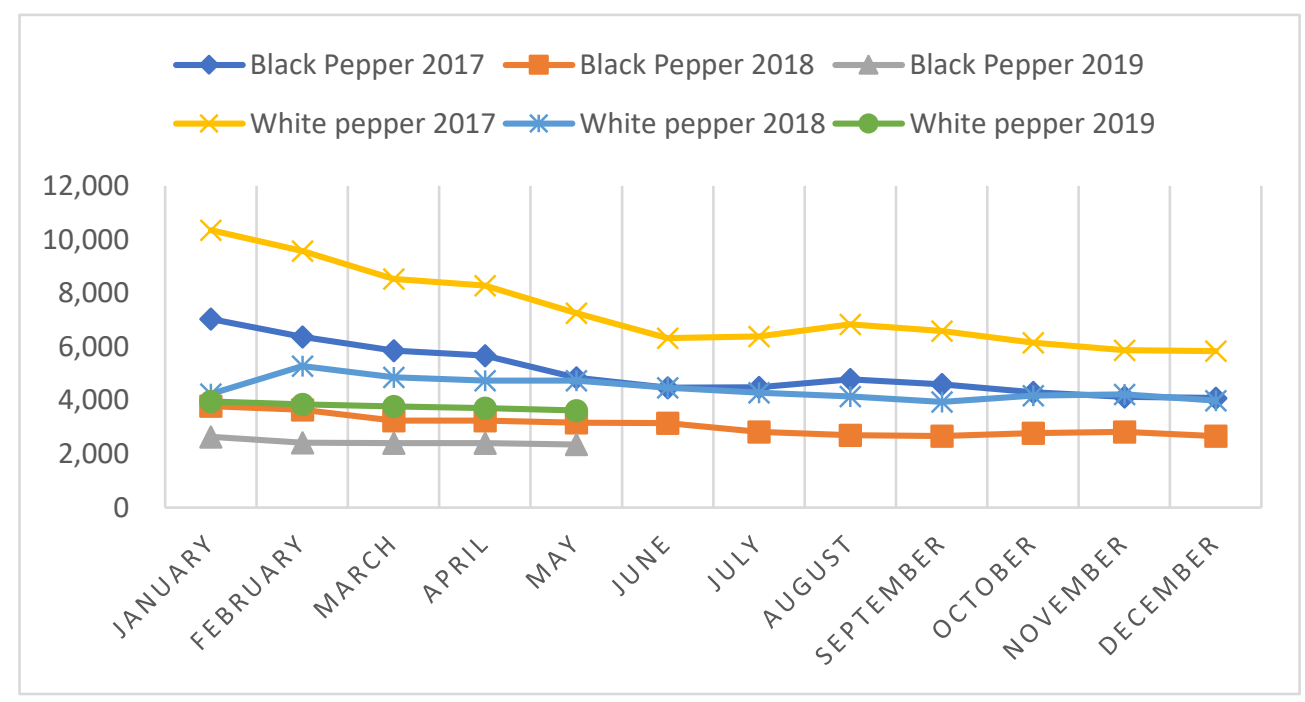

Figure 2. Composite price of black pepper and white pepper from 2017 to May 2019 (International Pepper Community, 2019).

Growth of black pepper veins requires proper fertilizer management along with their growing stages (Yap, 2012b) with good structure and water-holding capacity (Srinivasan et $a l .$, 2007). Cultivation of pepper farm majorly conducted by small farmers, rarely implement the usage of technology (Bermawie et al., 2019) and varies in term of soil, climatic conditions, demographic and socioeconomic (Paduit et al., 2018; Rosli et al., 2013). 
In terms of nutrient uptake, growth of black pepper vines are influenced by macronutrients such as N, P, K, Ca and Mg (Srinivasan et al., 2007; Yap, 2012a) and micronutrients such iron, zinc, manganese, molybdenum (Abd Hamid \& Wan Yahaya, 2019). However, the responses by each individual vine may varies depends on its varieties, environment, and biophysical characteristic of the area. According to Yap (2012b), the growth stages of black pepper can be differentiate into three stages (refer Figure 3). However, precise information on individual vein or even at different stages are still not being discovered. Hence, the needs for implementing geospatial technology is suggested in order to fulfil this gap.

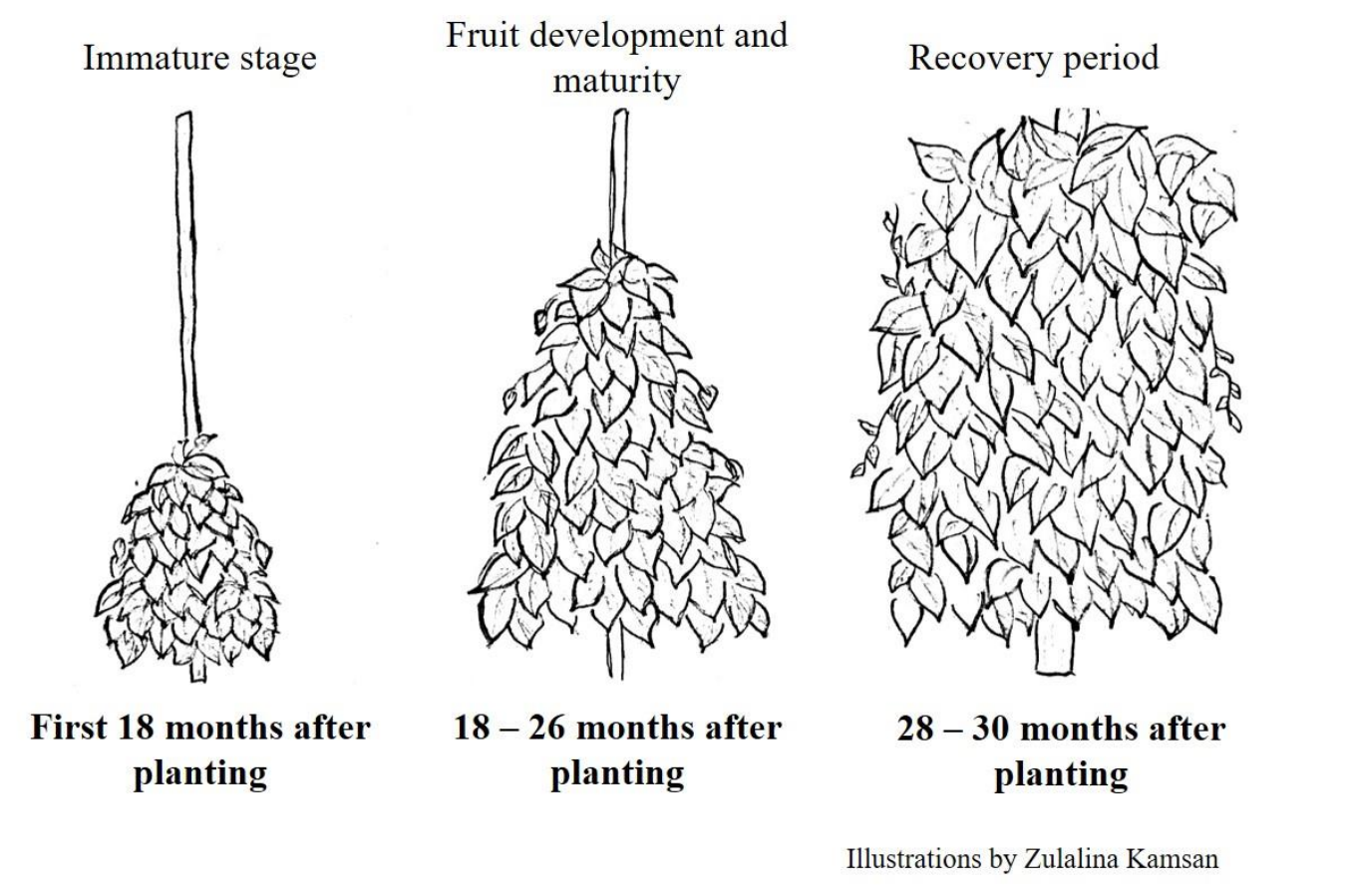

Figure 3. Growth stages of black pepper 30 months after planting. Illustration adapted from Yap (2012b).

Although the description for black pepper needs and requirements have been explained in general, detail information is scarce since the cultivation of this crop is varying. Improvement on black pepper cultivation that have been conducted until now is summarized in Table 1.

Table 1. Studies related with black pepper for cultivation amendment.

\begin{tabular}{cllll}
\hline & $\begin{array}{c}\text { Purpose of the } \\
\text { study }\end{array}$ & $\begin{array}{c}\text { Geospatial } \\
\text { instrument }\end{array}$ & Methods of Analysis & Study Gap \\
\hline $\begin{array}{clll}\text { (Kandiannan } \text { et } \\
\text { al., 2002) }\end{array}$ & $\begin{array}{l}\text { Identifying } \\
\text { method to } \\
\text { estimate }\end{array}$ & $\begin{array}{l}\text { Electronic leaf } \\
\text { area meter LI- }\end{array}$ & Allometric relationship & $\begin{array}{l}\text { Crop growth } \\
\text { condition status }\end{array}$ \\
& $\begin{array}{l}\text { individual leaf } \\
\text { area-efficient }\end{array}$ & COR) & Logarithm \\
transformation & & \\
& & & &
\end{tabular}




\begin{tabular}{|c|c|c|c|c|}
\hline & $\begin{array}{c}\text { Purpose of the } \\
\text { study }\end{array}$ & $\begin{array}{l}\text { Geospatial } \\
\text { instrument }\end{array}$ & Methods of Analysis & Study Gap \\
\hline (Yap, 2012b) & $\begin{array}{l}\text { Study the effect } \\
\text { of chemical and } \\
\text { organic } \\
\text { fertilizers on } \\
\text { some } \\
\text { physiological } \\
\text { characteristics, } \\
\text { yield and soil } \\
\text { fertility }\end{array}$ & $\begin{array}{l}\text { Leaf area meter } \\
\text { (CI - 202 Laser } \\
\text { Area Meter } \\
\text { Potable } \\
\text { photosynthesis } \\
\text { system (PPS } \\
\text { System, model } \\
\text { TPS 200) } \\
\text { Statistical } \\
\text { Package for } \\
\text { Social Science } \\
\text { (SPSS) }\end{array}$ & $\begin{array}{l}\text { Leaf area index } \\
\text { Chlorophyll content } \\
\text { Photosynthetic rate } \\
\text { Transpiration rate } \\
\text { One-way Analysis of } \\
\text { Variance (ANOVA) } \\
\text { Duncan Multiple } \\
\text { Ranged test(Yap, } \\
\text { 2012b) }\end{array}$ & $\begin{array}{l}\text { The nutrient } \\
\text { requirement at } \\
\text { different stages }\end{array}$ \\
\hline $\begin{array}{l}\text { (Hao et al., } \\
\text { 2012) }\end{array}$ & $\begin{array}{l}\text { Understanding } \\
\text { bioclimatic } \\
\text { distribution, } \\
\text { maximum } \\
\text { entropy based } \\
\text { on ecological } \\
\text { niche modelling } \\
\text { for black pepper } \\
\text { cultivation }\end{array}$ & $\begin{array}{l}\text { Maxent software } \\
\text { ver. } 2.3 \\
\text { GIS software } \\
\text { ArcView GIS } \\
\text { ver.3.2 }\end{array}$ & $\begin{array}{l}\text { Jackknife evaluation } \\
\text { Ecological Niche } \\
\text { Modelling (ENM) } \\
\text { WorldClim bioclimatic } \\
\text { data }\end{array}$ & $\begin{array}{l}\text { Preferable } \\
\text { ecology for black } \\
\text { pepper in } \\
\text { Malaysia }\end{array}$ \\
\hline $\begin{array}{c}\text { (Kho \& Shang, } \\
\text { 2016) }\end{array}$ & $\begin{array}{l}\text { Establishment } \\
\text { of new planting } \\
\text { material } \\
\text { cultivation } \\
\text { methods for } \\
\text { high production }\end{array}$ & - & $\begin{array}{l}\text { W-configuration } \\
\text { V-configuration } \\
\text { Single post or normal } \\
\text { planting } \\
\text { (ANOVA) by SPSS } \\
\text { Statistic Version } 16.0 \\
\text { ROI analysis }\end{array}$ & $\begin{array}{l}\text { New planting } \\
\text { management } \\
\text { requirement }\end{array}$ \\
\hline (Yap, 2016) & $\begin{array}{l}\text { Study the effect } \\
\text { of foliar } \\
\text { fertilizer when } \\
\text { used in } \\
\text { combination } \\
\text { with soil NPK } \\
\text { fertilizer on } \\
\text { different growth } \\
\text { parameters of } \\
\text { the Cultivar } \\
\text { Semongok } \\
\text { Aman }\end{array}$ & - & $\begin{array}{l}\text { Phytohormone analysis } \\
\text { free amino acids } \\
\text { analysis }\end{array}$ & $\begin{array}{l}\text { Specific amount } \\
\text { of fertilizer } \\
\text { required by single } \\
\text { vein }\end{array}$ \\
\hline $\begin{array}{c}\text { (Sulok et al., } \\
\text { 2018) }\end{array}$ & $\begin{array}{l}\text { Introducing } \\
\text { natural farming }\end{array}$ & - & - & $\begin{array}{l}\text { Soil and water } \\
\text { availability for } \\
\text { black pepper crop }\end{array}$ \\
\hline
\end{tabular}




\begin{tabular}{lclc}
\hline $\begin{array}{c}\text { Purpose of the } \\
\text { study }\end{array}$ & $\begin{array}{c}\text { Geospatial } \\
\text { instrument }\end{array}$ & Methods of Analysis & Study Gap \\
\hline $\begin{array}{l}\text { on black pepper } \\
\text { cultivation }\end{array}$ & & & \\
\hline
\end{tabular}

In conclusion, there are gaps of study in black pepper cultivation and management which involve nutrient requirements and uptake by black pepper vein. Therefore, introducing geospatial technology to monitor the growth and condition of the crops may enhance the black pepper cultivation and productivity.

\section{Geospatial Technology and Its Implementation in Malaysia}

Geospatial technology is one of the categorizations in precision agriculture where the integration of technologies such as geographical information system (GIS), global navigation satellite system (GNSS) and remote sensing, resulting in decision making through accurate and timely manner data collection and analysis (Martinez, 2017; Prim, 2017). Data sources of geospatial can be obtained as spatial product or spectral resolution (Rudiyanto et al., 2019). Analysis of geospatial application for agriculture sector may encompass biophysical characteristics, growth monitoring, yield forecasting and market prediction (Nurmiaty et al., 2019).

Demand for geospatial technology and their service in Malaysia are still at the implementation level where the adaptation focusing mostly on large scale cultivation area such as for oil palm and paddy. This precision technology carried out activities which used sensor system and variable rate nutrient for crop, soil, pest diseases and water management (Geospatial Media and Communication, 2015). Therefore, by implementing the geospatial application in black pepper may improve black pepper productivity regardless of their variation in land agroecology and climate. Besides, the analysis may encompass biophysical characteristics, growth monitoring, yield forecasting and market prediction (Nurmiaty et al., 2019).

\section{Crop Growth Monitoring System (CGMS) Based on Geospatial Application}

Nowadays, monitoring of crop growth using the integration of geospatial based technologies have started to arise. The systems are implemented both in macro (such as mapping, surveying, land use and land cover classification) and micro levels of agricultural activities (such as mapping of ground water resources, drainage pattern, variable rate application and input management) (Geospatial Media and Communication, 2015; Chong et al., 2017). A framework to establish CGMS is described by Clevers et al., (1994) which focusing on integrating optical remote sensing data from various sources to estimate the parameters such as leaf area index (LAI), leaf angle distribution (LAD), and leaf colour (optical properties in the photosynthetically active radiation (PAR) region). Table 2 presents detailed system of CGMS that have been developed around the world. Global Information 
and Early Warning System (GIEWS) is meant to be the first crop growth monitoring model developed and provided information on food production. Modification of certain system is done to establish other monitoring systems based on specific region and crops interest.

Table 2. Crop growth monitoring systems around the world.

\begin{tabular}{|c|c|c|c|}
\hline System & $\begin{array}{c}\text { Year of } \\
\text { establishing }\end{array}$ & Input/Parameter & Output of system \\
\hline $\begin{array}{l}\text { Global Information and } \\
\text { Early Warning System } \\
\text { (GIEWS) (FAO, n.d.) }\end{array}$ & 1970 & $\begin{array}{ll}\text { - } & \text { Auxiliary data } \\
\text { - } & \text { Rainfall estimate } \\
\text { - } & \text { NDVI } \\
\text { - } & \text { Agriculture stress index (ASI) } \\
& 2013\end{array}$ & \begin{tabular}{ll} 
- & \multicolumn{2}{l}{ Regular bulletin } \\
- & Market on global \\
& scale \\
- & Specific regional \\
report
\end{tabular} \\
\hline $\begin{array}{l}\text { Famine Early Warning } \\
\text { System Network } \\
\text { (FEWSNET) } \\
\text { (Funk \& Verdin, 2009) }\end{array}$ & 1985 & $\begin{array}{ll}\text { - } & \text { Agro-climatology data } \\
\text { - } & \text { Field assessment } \\
\text { - } & \text { Market price monitoring } \\
\text { - } & \text { Nutrition surveillance conflicts }\end{array}$ & $\begin{array}{l}\text { - Decision support to } \\
\text { food assistance } \\
\text { programs } \\
\text { - Quantify chances in } \\
\text { planting area with } \\
\text { crop yield }\end{array}$ \\
\hline $\begin{array}{l}\text { World Food Studies } \\
\text { (WOFOST) } \\
\text { (Diepen \& Wit, 2014) }\end{array}$ & 1986 & $\begin{array}{ll}\text { - } & \text { Weather data } \\
\text { - } & \text { Crop data } \\
\text { - } & \text { Soil water balance data }\end{array}$ & $\begin{array}{l}\text { - Growth and crop } \\
\text { production analysis }\end{array}$ \\
\hline $\begin{array}{l}\text { Monitoring Agriculture } \\
\text { with Remote Sensing } \\
\text { (MARS) Crop Yield } \\
\text { Forecasting System } \\
\text { (MCYFS) (Boogard, et } \\
\text { al., 2007) }\end{array}$ & 1992 & $\begin{array}{ll}\text { - } & \text { Weather data } \\
\text { - } & \text { Crop model simulation } \\
\text { - } & \text { Biophysical parameter }\end{array}$ & $\begin{array}{ll}\text { - } & \text { Operational estimate } \\
\text { area } \\
\text { - } \\
\text { Yield and } \\
\text { production } \\
\text { - Independent } \\
\text { evidence } \\
\text { - Crop maps }\end{array}$ \\
\hline $\begin{array}{c}\text { Crop watch } \\
\text { (Wu et al., 2015) }\end{array}$ & 1998 & $\begin{array}{l}\text { - } \text { Global } \\
\text { - } \text { Rainfall, temperature, } \\
\text { photosynthetically active } \\
\text { radiation, potential biomass } \\
\text { - } \text { Regional } \\
\text { - } \text { Vegetation health index, } \\
\text { vegetation condition index } \\
\text { - } \\
\text { - } \\
\text { Dational / Sub - regional }\end{array}$ & $\begin{array}{l}\text { - Timely, reliable, } \\
\text { independent } \\
\text { prediction of crops } \\
\text { condition and } \\
\text { production within } \\
\text { China and global }\end{array}$ \\
\hline $\begin{array}{l}\text { United States } \\
\text { Department of }\end{array}$ & 2001 & $\begin{array}{l}\text { - Extraction and process of } \\
\text { agrometeorological data }\end{array}$ & $\begin{array}{l}\text { - Prediction of global } \\
\text { crop production }\end{array}$ \\
\hline Agriculture — Foreign & & - Visualize data from & - Market intelligence \\
\hline $\begin{array}{l}\text { Agriculture Services } \\
\text { (USDA - FAS) } \\
\text { (Baruth } \text { et al., 2008) }\end{array}$ & & $\begin{array}{l}\text { TOPEX/Poseidon Jason - } 1 \\
\text { Satellite } \\
\text { - } \text { GLAM Project (2002) } \\
\text { - Semi - automated classification } \\
\text { algorithm and remote sensing }\end{array}$ & \\
\hline $\begin{array}{l}\text { China Agriculture } \\
\text { Remote Sensing } \\
\text { Monitoring System } \\
\text { (CHARMS) }\end{array}$ & 2001 & $\begin{array}{l}\text { - } \text { Weather data } \\
\text { - } \quad \text { Crop characteristics data } \\
\text { - } \quad \text { Soil information data } \\
\text { - }\end{array}$ & $\begin{array}{l}\text { - Crop growth } \\
\text { condition at different } \\
\text { level of aggregation }\end{array}$ \\
\hline
\end{tabular}




\begin{tabular}{|c|c|c|c|}
\hline System & $\begin{array}{c}\text { Year of } \\
\text { establishing }\end{array}$ & Input/Parameter & Output of system \\
\hline (Huang et al., 2011) & & $\begin{array}{ll}\text { - } & \text { Administrative region vector data } \\
\text { - } & \text { Agro - meteorological modelling } \\
\text { - } & \text { Statistical analysis tools }\end{array}$ & \\
\hline $\begin{array}{c}\text { GEOGLAM } \\
\text { (Fritz et al., 2019) }\end{array}$ & 2011 & $\begin{array}{ll}- & \text { Crop growing conditions } \\
- & \text { Crop status } \\
\text { - } & \text { Agro - climatic conditions }\end{array}$ & $\begin{array}{l}\text { - Agricultural market } \\
\text { assessment system }\end{array}$ \\
\hline $\begin{array}{l}\text { World Food Program } \\
\text { Seasonal Monitor } \\
\text { (Fritz et al., 2019) }\end{array}$ & 2014 & $\begin{array}{ll}\text { - } & \text { Near real - time rainfall estimate } \\
\text { (CHIRPS) } \\
\text { - } & \text { NDVI - MODIS } \\
\text { - } & \text { Anomalis of parameters } \\
\text { - } & \text { Seasonal explorer data } \\
\text { - } & \text { Price forecast information }\end{array}$ & 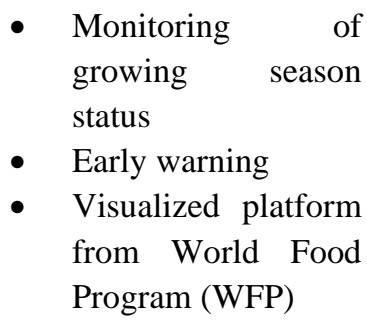 \\
\hline $\begin{array}{l}\text { Anomally HotSpots of } \\
\text { Agriculture Production } \\
\text { (ASAP) (Rembold et al., } \\
2017)\end{array}$ & 2017 & $\begin{array}{ll}\text { - } & \text { Rainfall estimation } \\
\text { - } & \text { NDVI - Remote sensing } \\
\text { - } & \text { Agricultural Monitoring Experts } \\
\text { - } & \text { Earth observation analysis } \\
\text { Weather data }\end{array}$ & $\begin{array}{l}\text { Unfavourable } \\
\text { growing area } \\
\text { identification for } \\
\text { crops and rangeland }\end{array}$ \\
\hline
\end{tabular}

\subsection{Remote Sensing-Based Mapping}

Mapping using remote sensing is one of the essential fundamental for crop growth monitoring. The component helps in producing accurate crop maps throughout their growing season (Li \& Chen, 2011; Song et al., 2017; Sousa \& Small, 2019) hence reassure for precise decision making. Recently, increase application of remotely sensed data for monitoring altered by the availability of open data, advanced machine learning methods, and access to cloud computing platforms to handle big data storage and processing (Kussul et al., 2018; Rudiyanto et al., 2019). Apart from that, these methods also provide a cost-effective and site-specific assessment for monitoring the crop growth condition in real-time (Carolita et al., 2018).

Satellite data sources for mapping can be obtained through optical product (Landsat, Sentinel-2, MODIS, RapidEye, SPOT and PROB-V) and synthetic aperture radar (SAR) or microwave product (Sentinel-1, RADARSAT) (Rudiyanto et al., 2019). Optical systems measure reflected sunlight and provide spectral properties of their targets. This system is known to be susceptible to the environment especially on cloud cover which resulting in difficulties for image classification and discrimination (Tan et al., 2012; Kussul et al., 2018). Whilst, SAR or microwave data are more stable towards atmosphere factors. As mentioned by Zainol et al., (2019) feasible use of hyperspectral sensing for discriminating vegetation species and its potential use for habitat mapping .

However, selection of this method requires a better understanding of the complex relationship between the object and sensor parameter such as frequency, polarization setting and incidence angle (Yuzugullu et al., 2017). Images data were collected through the 
reflection of (red, green, blue and near-infrared, NIR) wavelength (Fieuzal et al., 2016). Berhane et al., (2018) approach mentioned that, combination of few nonparametric classifiers such as decision-tree-rule-based (RB), random forest (RF) can be coupled to improve overall accuracy (OA) of the image results produced. Table 3 shows satellite types that are commonly used in agricultural industry.

Table 3. Types of satellite commonly used for agriculture studies.

\begin{tabular}{|c|c|c|c|c|}
\hline $\begin{array}{l}\text { Types of } \\
\text { satellite }\end{array}$ & $\begin{array}{c}\text { Launch } \\
\text { year }\end{array}$ & Resolution & Revisit cycle & Spectral band $(\mu \mathrm{m})$ \\
\hline $\begin{array}{c}\text { Landsat } \\
\text { 7ETM }\end{array}$ & 1999 & $15-60 \mathrm{~m}$ & 16 days & $\begin{array}{l}\text { Blue (0.44-0.5) Green }(0.52-0.60) \text { Red } \\
(0.63-0.69) \text { NIR }(0.77-0.90) \text { SWIR } 1 \\
(1.55-1.75) \text { TIR }(10.31-12.36) \text { SWIR } 2 \\
(2.06-2.35) \text { Pan }(0.52-0.90)\end{array}$ \\
\hline MODIS & 2000 & $500 \mathrm{~m}$ & 1-2 days & $\begin{array}{l}\text { *Bands are depending on its primary } \\
\text { use. Available bands: } \\
1(620-670), 2(841-876), 3(459-479) \\
4(545-565), 5(1230-1250), 6(1628 \\
1652), 7(2105-2155), 20(3.66-3.840) \\
21(3.929-3.989), 22(3.929-3.989), 23 \\
(4.020-4.080), 31(10.780-11.280), 32 \\
(11.770-12.270)\end{array}$ \\
\hline SPOT 5 & 2002 & $5-20 \mathrm{~m}$ & $\begin{array}{l}\text { 2-3 days, } \\
\text { depending on } \\
\text { latitude }\end{array}$ & $\begin{array}{l}\text { Red }(610-680) \text { NIR }(780-890) \text { SWIR } \\
(1,580-1,750)\end{array}$ \\
\hline $\begin{array}{l}\text { RapidEye } \\
\quad(5 \mathrm{~m})\end{array}$ & 2008 & $5 \mathrm{~m}$ & $\begin{array}{l}\text { Daily (off- } \\
\text { nadir)/5.5 days } \\
\text { (at nadir) }\end{array}$ & $\begin{array}{l}\text { Blue }(0.44-0.51) \text { Green }(0.52-0.59) \text { Red } \\
(0.63-0.69) \text { Red Edge }(0.69-0.73) \text { NIR } \\
(0.76-0.85)\end{array}$ \\
\hline SPOT 6 & 2012 & $\begin{array}{c}1.5 \mathrm{~m} \\
(5-10 \mathrm{~m} \\
\text { accuracy })\end{array}$ & $\begin{array}{l}\text { Provide option of } \\
\text { daily revisit }\end{array}$ & $\begin{array}{l}\text { Blue }(0.45-0.52) \text { Green }(0.53-0.59) \text { Red } \\
(0.63-0.70) \text { NIR }(0.76-0.90)\end{array}$ \\
\hline Landsat 8 & 2013 & $15-30 \mathrm{~m}$ & $\begin{array}{l}\text { Circle the earth } \\
\text { every } 98.9 \\
\text { minutes }\end{array}$ & $\begin{array}{l}\text { Blue }(0.45-0.51) \text { Green }(0.53-0.59) \text { Red } \\
(0.64-0.67) \text { NIR }(0.85-0.88) \text { SWIR } 1 \\
(1.57-1.65) \text { TIR } 1 \quad(10.6-11.2) \text { TIR } 2 \\
(11.5-12.5) \text { SWIR } 2(2.11-2.29) \text { Cirrus } \\
(1.36-1.38) \text { Pan }(0.50-0.68)\end{array}$ \\
\hline $\begin{array}{l}\text { Project for } \\
\text { on Board } \\
\text { Autonomy } \\
\text { (PROBA-V) }\end{array}$ & 2013 & $\begin{array}{c}1 \mathrm{~km}-300 \\
\mathrm{~m}\end{array}$ & $\begin{array}{l}90 \% \text { daily } \\
\text { coverage of } \\
\text { equatorial zones } \\
\text { and } 100 \% \text { two- } \\
\text { daily imaging }\end{array}$ & $\begin{array}{l}\text { Blue (0.46; FWHM 0.042) Red (CWL } \\
0.66 ; \text { FWHM 0.082) NIR (0.83; FWHM } \\
0.012) \text { SWIR (1.61; FWHM 0.089) }\end{array}$ \\
\hline Sentinel-2 & 2015 & & $\begin{array}{l}10 \text { days at the } \\
\text { equator with one } \\
\text { satellite, five } \\
\text { days with two } \\
\text { satellite }\end{array}$ & $\begin{array}{l}\text { Coastal }(0.442) \text { Blue }(0.492) \text { Green } \\
(0.559) \text { Red }(0.665) \text { Vege Red }(0.704) \\
\text { Vege Red }(0.739) \text { Vege Red }(0.780) \text { NIR } \\
(0.833) \text { Narrow NIR (0.864) Water } \\
\text { Vapour }(0.943) \text { SWIR Cirrus } 1.377 \\
\text { SWIR (1.610) SWIR }(2.186)\end{array}$ \\
\hline
\end{tabular}

(NIR — Near Infrared, SWIR — Shortwave Infrared, CWL Central wavelength, FWHM — Full width at half maximum). 
Therefore, in monitoring black pepper growth and condition, mapping based on satellite might give advantages in reducing cost and provide more accurate real-time data since this crop are labour dependent. However, to obtain clear and concise data from remote sensing sometimes withstanding issues like clouds cover and low spectral resolution which require expertise to handle such situation.

\subsection{Unmanned Aerial Vehicle (UAV) based Mapping}

Unmanned aerial vehicles (UAV) have been used for range of purposes including soil sampling, irrigation management, precision spraying, mechanical weeding, and crop harvesting (Bonadies et al., 2016; Mat Su et al., 2017). Apart from remote sensing based satellite images, UAV started to get attention among agricultural practitioner considering their capabilities to monitor (Chang et al., 2017) and capture clearer image at specific height (Grenzdörffer, 2014) without the disturbance of cloud cover. Besides that, classification methods from UAV images may exhibits the effect of salt and pepper of the data (Chen et al., 2019). Despite its capabilities, this UAV are rarely being implemented on small scale cultivation since it requires high investment and skills to handle this technology. Hence, a better approach needs to be strategized either by the authority or responsible organization in order to enhance the application of UAV on small scale cultivation.

\subsection{Monitoring Assessment}

To improve crops productivity, farmers need to have detailed understandings on crop growth status during specific development stage throughout the growing season (Du \& Noguchi, 2017). Using geospatial technology, monitoring of black pepper might help in reducing cost of production since black pepper are recorded as one of the commodity crops with high production cost issues (Kamarudin et al., 2013; Paduit et al., 2018). Detection of individual crops change based on variation of colour through satellite image (Martinez, 2017) may help to identify neither nutrient requirement nor pest or disease infection on individual vines hence fill in the gap on black pepper total nutrient uptake and distribution in different parts (Paduit et al., 2018).

Analyzation of vegetation indices calculation such as normalized difference vegetation index (NDVI), leaf area index (LAI), ratio vegetation index (RVI), and perpendicular vegetation index (PVI) (Panda et al., 2010; Martinez, 2017; Xue \& Su, 2017) will derive an equation obtained from different shades of colour obtained from the images thus resulting an information for decision making (Raeva et al., 2019). Through the model, growth monitoring activity might grow and successful of each model will lead towards a better system as grown in other developed countries (Sharifi, 2000; Meng et al., 2006; Huang et al., 2011). The difference in reflected waves of different green leaves and crops canopy enable various analysis such as crop classification, pest disease detection and growing pattern of the crop from time to time. In Malaysia, monitoring of crops using geospatial only focus mostly on rice (refer Table 4) and large-scale cultivation commodity crops such as oil palm. 
Hence, implementing geospatial application for black pepper are expected to show progression for the crops and arouse improvement on other small-scale cultivation in Malaysia.

Table 4. Geospatial application for crop monitoring.

\begin{tabular}{|c|c|c|c|c|c|}
\hline & $\begin{array}{c}\text { Area of } \\
\text { study }\end{array}$ & $\begin{array}{c}\text { Purpose of } \\
\text { study }\end{array}$ & $\begin{array}{c}\text { Subject of } \\
\text { Study }\end{array}$ & $\begin{array}{l}\text { Geospatial } \\
\text { instrument }\end{array}$ & $\begin{array}{c}\text { Methods of } \\
\text { Analysis }\end{array}$ \\
\hline $\begin{array}{l}\text { (Sousa } \text { et } \\
\text { al., 2019) }\end{array}$ & $\begin{array}{l}\text { Mapping and } \\
\text { monitoring }\end{array}$ & $\begin{array}{l}\text { Mapping and } \\
\text { monitoring using } \\
\text { optical and } \\
\text { thermal } \\
\text { measurement }\end{array}$ & Rice & $\begin{array}{c}\text { Landsat } 4-8 \text { image } \\
\text { time series. }\end{array}$ & $\begin{array}{l}\text { - Spatiotemporal } \\
\text { characterization } \\
\text { - Temporal } \\
\text { mixture } \\
\text { modelling }\end{array}$ \\
\hline $\begin{array}{l}\text { (Franch et } \\
\text { al., 2019) }\end{array}$ & Forecasting & $\begin{array}{l}\text { Developing new } \\
\text { crop model based } \\
\text { on Difference } \\
\text { Vegetation Index } \\
\text { (DVI) extracted } \\
\text { from MODIS } \\
\text { data }\end{array}$ & Winter wheat & MODIS & - $\quad(\mathrm{DVI})$ \\
\hline $\begin{array}{c}\text { (Defourny et } \\
a l ., 2019)\end{array}$ & Monitoring & $\begin{array}{l}\text { Propose methods } \\
\text { and develop an } \\
\text { open source } \\
\text { system suitable } \\
\text { for most } \\
\text { cropping } \\
\text { systems. }\end{array}$ & $\begin{array}{l}\text { Maize } \\
\text { Vegetables } \\
\text { Wheat } \\
\text { Sunflower } \\
\text { Rapeseed } \\
\text { Soybean } \\
\text { Rice } \\
\text { Cassava } \\
\text { wheat-oat } \\
\text { Sorghum } \\
\text { Millet } \\
\text { Sesame } \\
\text { Cotton } \\
\text { Barley } \\
\text { Fodder crops } \\
\text { Oilseed crops }\end{array}$ & $\begin{array}{l}\text { Copernicus } \\
\text { Sentinel-2 }\end{array}$ & Sen2-Agri system \\
\hline $\begin{array}{l}\text { (Li et al., } \\
\text { 2019) }\end{array}$ & $\begin{array}{l}\text { Monitoring } \\
\text { and } \\
\text { classification }\end{array}$ & $\begin{array}{l}\text { Exploring the } \\
\text { potential of L- } \\
\text { band fully } \\
\text { polarimetric } \\
\text { UAVSAR for } \\
\text { monitoring and } \\
\text { classification of } \\
\text { crops }\end{array}$ & $\begin{array}{l}\text { Almond } \\
\text { Walnut } \\
\text { Alfalfa } \\
\text { Winter wheat } \\
\text { Corn } \\
\text { Sunflower } \\
\text { tomato }\end{array}$ & $\begin{array}{l}\text { Uninhabited Aerial } \\
\text { Vehicle Synthetic } \\
\text { Aperture Radar } \\
\text { (UAVSAR) }\end{array}$ & $\begin{array}{ll}\cdot \quad \text { Linear } \\
\text { polarization } \\
\text { (HH, HV, VV) } \\
\text { Polarimetric } \\
\text { decomposition } \\
\text { (Cloude-Pottier } \\
\& \text { Freeman- } \\
\text { Durden) }\end{array}$ \\
\hline $\begin{array}{l}*(\text { Rudiyanto } \\
\text { et al., 2019) }\end{array}$ & $\begin{array}{l}\text { Mapping, } \\
\text { monitoring } \\
\text { and } \\
\text { phenology } \\
\text { study }\end{array}$ & $\begin{array}{l}\text { Mapping and } \\
\text { monitoring of } \\
\text { rice growth } \\
\text { extent and } \\
\text { cropping patterns } \\
\text { over a large area. }\end{array}$ & Rice & $\begin{array}{l}\text { Temporal } \\
\text { Sentinel-1 } \\
\text { Google Earth } \\
\text { Engine (GEE) } \\
\text { cloud-based } \\
\text { platform. }\end{array}$ & $\begin{array}{ll}\text { - } & \text { K-means } \\
\text { clustering } \\
\text { - } & \text { Hierarchical } \\
\text { cluster analysis } \\
\text { (HCA) } \\
\text {. } \\
\text { Visual } \\
\text { interpretation } \\
\text { of } \quad \text { VH } \\
\text { polarization }\end{array}$ \\
\hline
\end{tabular}




\begin{tabular}{|c|c|c|c|c|c|}
\hline & $\begin{array}{l}\text { Area of } \\
\text { study }\end{array}$ & $\begin{array}{c}\text { Purpose of } \\
\text { study }\end{array}$ & $\begin{array}{l}\text { Subject of } \\
\text { Study }\end{array}$ & $\begin{array}{l}\text { Geospatial } \\
\text { instrument }\end{array}$ & $\begin{array}{l}\text { Methods of } \\
\text { Analysis }\end{array}$ \\
\hline $\begin{array}{l}\text { (Kern et al., } \\
\text { 2018) }\end{array}$ & Forecasting & $\begin{array}{l}\text { Yield simulation } \\
\text { using multiple } \\
\text { linear regression } \\
\text { model }\end{array}$ & $\begin{array}{l}\text { Winter wheat } \\
\text { Rapeseed } \\
\text { Maize } \\
\text { sunflower }\end{array}$ & $\begin{array}{l}\text { - Open Database } \\
\text { for Climate } \\
\text { Change Related } \\
\text { - Impact Studies } \\
\text { in Central } \\
\text { Europe } \\
\text { (FORESEE) } \\
\text { - Medium-Range } \\
\text { Weather } \\
\text { Forecast } \\
\text { (ECMWF) } \\
\text { ERA-Interim } \\
\text { database } \\
\text { MODIS sensor } \\
\text { on Terra } \\
\text { satellite }\end{array}$ & 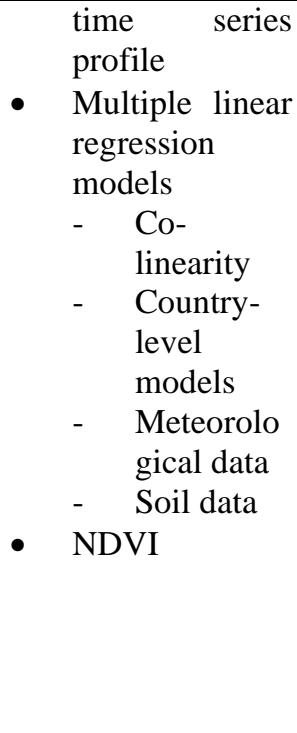 \\
\hline $\begin{array}{c}*(\text { Che Ya et } \\
\text { al., 2018) }\end{array}$ & Monitoring & $\begin{array}{l}\text { Early stage of } \\
\text { crop monitoring }\end{array}$ & Rice & $\begin{array}{ll}\text { - } & \text { RGB digital } \\
\text { camera } \\
\text { - } \\
\text { Multirotor } \\
\text { UAV }\end{array}$ & - $\quad$ Spatial analysis \\
\hline $\begin{array}{c}*(\text { Abd } \\
\text { Hamid et } \\
\text { al., 2017) }\end{array}$ & Monitoring & $\begin{array}{l}\text { Identification of } \\
\text { spatial } \\
\text { distribution of } \mathrm{N}, \\
\mathrm{P} \text {, and } \mathrm{K} \text { at two } \\
\text { pepper farms } \\
\text { planted in hilly } \\
\text { topography }\end{array}$ & Black pepper & - $\quad$ ArcGIS & $\begin{array}{ll}\text { - } & \text { Conceivable } \\
\text { correlation } \\
\text { - } \\
\text { Kriging } \\
\text { interpolation } \\
\text { method }\end{array}$ \\
\hline $\begin{array}{c}\text { (Carolita } e t \\
\text { al., 2018) }\end{array}$ & $\begin{array}{l}\text { Monitoring } \\
\text { and } \\
\text { phenology } \\
\text { study }\end{array}$ & $\begin{array}{l}\text { Growth profile } \\
\text { analysis to } \\
\text { estimate } \\
\text { productivity age } \\
\text { of oil palm }\end{array}$ & Oil Palm & - $\quad$ SPOT 6 & $\begin{array}{ll}- & \text { NDVI analysis } \\
\text { - } & \text { Regression } \\
& \text { analysis }\end{array}$ \\
\hline $\begin{array}{c}*(\text { Mohd } \\
\text { Hazir \& } \\
\text { Tuan Muda, } \\
\text { 2018) }\end{array}$ & $\begin{array}{l}\text { Mapping and } \\
\text { classification }\end{array}$ & $\begin{array}{l}\text { Viability } \\
\text { determination of } \\
\text { spectral analysis } \\
\text { to detect rubber } \\
\text { smallholdings } \\
\text { using satellite } \\
\text { imagery }\end{array}$ & Rubber & - $\quad$ Landsat 8 OLI & $\begin{array}{ll}\text { - } & \text { False Colour } \\
\text { - } & \text { Unethod } \\
& \text { Classification } \\
\text { - } & \text { Supervised } \\
\text { - } & \text { Classification } \\
\text { Twenty-seven } \\
\text { Spectral } \\
\text { Vegetation } \\
\text { Indices (SVIs) }\end{array}$ \\
\hline $\begin{array}{l}\text { (Chang et } \\
\text { al., 2017) }\end{array}$ & Monitoring & $\begin{array}{l}\text { Monitoring of } \\
\text { Sorghum } \\
\text { (Sorghum } \\
\text { bicolor) using an } \\
\text { Unmanned } \\
\text { Aerial System } \\
\text { (UAS) }\end{array}$ & Sorghum & $\begin{array}{l}\text { - UAV-DJI } \\
\text { Phantom } 2 \\
\quad \text { vision } \\
\text { platform, 14 } \\
\text { Ground control } \\
\text { point (GCP) } \\
\text { Real-time } \\
\text { kinematic } \\
\text { (RTK) GPS } \\
\text { unit }\end{array}$ & $\begin{array}{ll}\text { - } & \text { Processing of } \\
\text { UAS data } & \\
-\quad & \text { Digital } \\
& \text { Terrain } \\
& \text { Model } \\
& \text { (DTM) } \\
- & \text { Digital } \\
& \text { Surface } \\
& \text { Model } \\
& \text { (DSM) }\end{array}$ \\
\hline
\end{tabular}




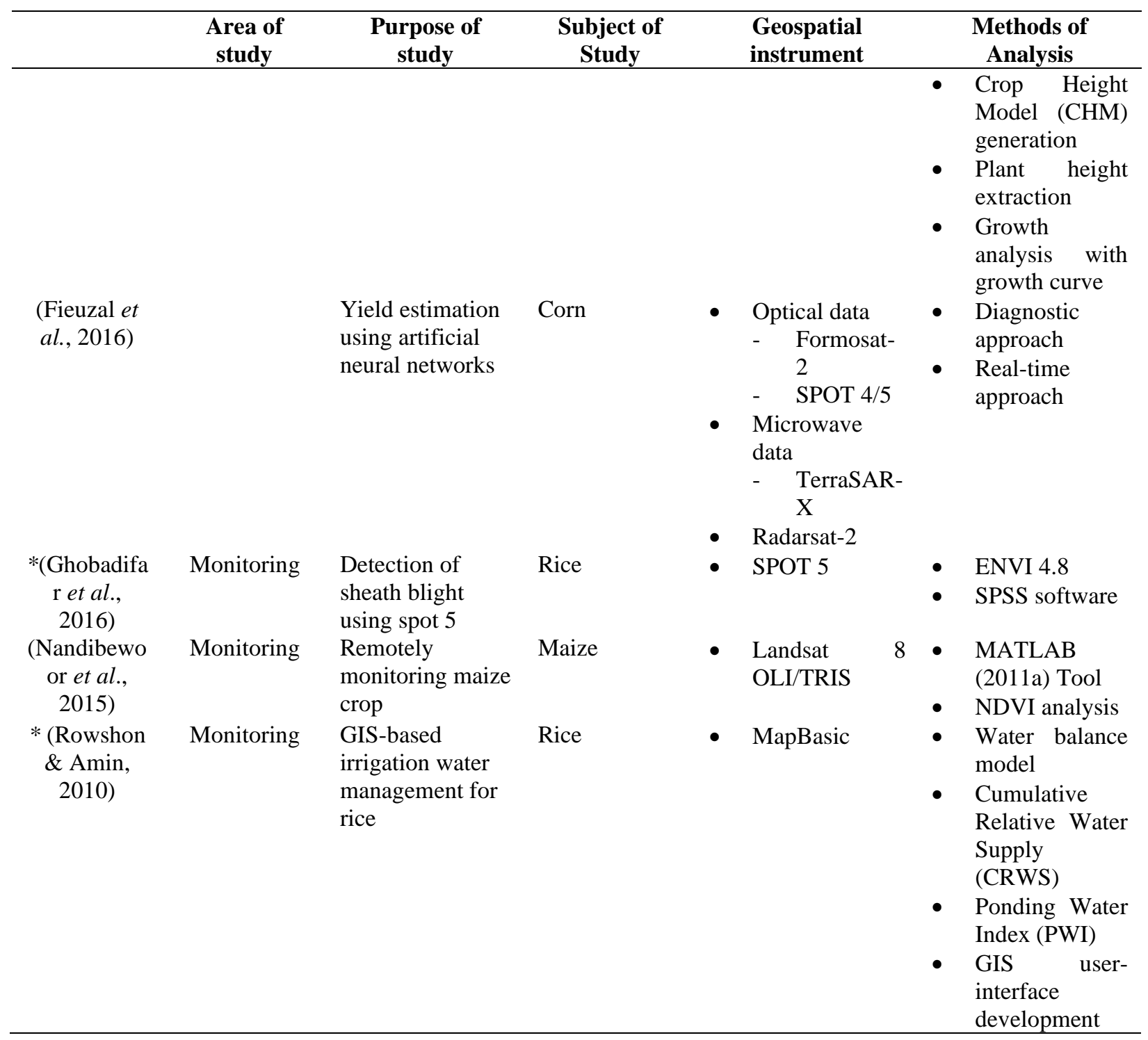

* Indicates the study was conducted in Malaysia.

\section{Limitations to Implement Geospatial Technology on Black Pepper Cultivation}

Earth observation (EO) has now considered as easily accessible data since the data can be retrieved freely (Kussul et al., 2018). Reliable and accurate crop classification maps are an important data source for agricultural monitoring and food security assessment studies (Orynbaikyzy et al., 2019). Anyhow, to obtain reliable data for small scale cultivation such as black pepper is challenging since the activities are done by small farmers and their location are mostly not a hot spot in order to be recorded frequently. Apart from that, geospatial technology requires specialists in order to run the analysis especially during implementation level yet, Malaysia is still not enough of expertise in this industry. Government policies are known to be one of the important drivers of technology utilization (Geospatial Media and Communication, 2015). Thereupon, both public and private agencies are needed in order to enhance the growth of this technology to be adapt in small scale crop cultivation. 
Next, black pepper in Malaysia mostly being planted in the hilly area where soil erosions are common especially during raining seasons. Thus, in order to collect ground data, installation of durable equipment with well establish shelf life are preferred to sustain data transmission for the cultivation area. This might lead to add in cost since high quality equipment are preferred. As mentioned by Rosli et al. (2013), circumstances that affect the implementation of technology in black pepper cultivation are education level, farming experiences and total of pepper vines. Thence, it may be tough to convince farmers to apply geospatial for their crops improvement. Again, government and agencies play a role to convey intensive agriculture extension and support in order to ensure consummation of geospatial technology for black pepper cultivation.

\section{Conclusion}

In conclusion, geospatial technology is proved to improve crop monitoring, yield estimation and productivity. However, their implementation should be expended for small scale cultivation such as black pepper since these crops are profitable commodity crops for most farmers in Malaysia. To attain this technology implementation for small scale area, various efforts need to consolidate. Efforts such as enhancing farmers knowledge on geospatial technology as well as proper management practice on black pepper is one of the essential. Next, cooperation between farmers, government and private sector is essentially needed. Thus, black pepper farmers could enhance their crop productivity and sustain Malaysia's black pepper quality.

This study is based on comprehensive study focusing only on few relatable studies on geospatial technology and black pepper cultivation since there are limitations on black pepper study especially using geospatial technology. Therefore, consistent study is suggested to improve black pepper productivity; especially for small scale cultivation in the future.

Author Contributions: Siti Zul Lailee Kamsan; writing_original draft preparation, Wan Nor Zanariah Zainol@Abdullah; writing_review and editing.

Funding: No external funding was provided for this research.

Acknowledgments: In this segment, you may acknowledge any support that is not addressed by the author's contribution or funding sections.

Conflicts of Interest: The authors declare no conflict of interest.

\section{References}

Abraham, A. (2018) .The trend in export, import and production performance of black pepper in India. International Journal of Pure and Applied Mathematics, 118 (18 Special), 4795-4800.

Aziemah, J. N. et al. (2017). Peluang dan potensi tanaman lada hitam di Sabah : Persepsi penerimaan penduduk daerah Tenom. e-Academia Journal, 6(2), 1-191.

Baruth, B. et al. (2008). The use of Remote sensing within the MARS crop yield monitoring system of the European commission. The International Archives of the Photogrammetry, Remote Sensing and 
Spatial Information Sciences.2, XXXVII. $\quad$ Retrieved from https://www.isprs.org/proceedings/XXXVII/congress/8_pdf/10_WG-VIII-10/02.pdf.

Berhane, T. M. et al. (2018). Decision-tree, rule-based, and random forest classification of high-resolution multispectral imagery for wetland mapping and inventory. Remote Sensing, 10(4). doi: $10.3390 / \mathrm{rs} 10040580$.

Bermawie, N. et al. (2019). Morphological characteristics, yield and quality of black pepper Ciinten variety in three agro ecological conditions. International Conference on Food Science \& Technology, 292(1). doi: 10.1088/1755-1315/292/1/012065.

Bonadies, S., Lefcourt, A. \& Gadsden, S. A. (2016). A survey of unmanneed ground vehicle with application to agricultural and environmental sensing. Autonomous Air and Ground Sensing Systems for Agricultural Optimization and Phenotyping. SPIE. doi: 10.1117/12.2224248.

Boogard, H., Roller, J. A. te \& Diepen, K. van (2007). In B. Baruth, G. Genovese, and O. Leo (Eds) CGMS Version 9.2 User Manual and technical documentation. Italy: European Communities.

Carolita, I. et al. (2018). Growth profile analysis of oil palm by using spot 6 the case of North Sumatra. International Journal of Remote Sensing and Earth Sciences (IJReSES), 12(1), 21. doi: 10.30536/j.ijreses.2015.v12.a2669.

Chang, A. et al. (2017). Crop height monitoring with digital imagery from Unmanned Aerial System (UAS). Computers and Electronics in Agriculture2, 141, 232-237. doi: 0168-1699.

Chao-yun, H. A. O. et al. (2012). Modeling the potential geographic distribution of black pepper (Piper nigrum) in Asia Using GIS Tools', Journal of Integrative Agriculture, 11(4), pp. 593-599. doi: 10.1016/S20953119(12)60046-X.

Charyulu, K. R. C. et al. (2019). Case studies on the utilization of geospatial technology for sustainable agriculture. International Journal of Current Microbiology and Applied Sciences, 8(03), 112-120. doi: 10.20546/ijcmas.2019.803.016.

Chen, L. et al. (2019). Water requirement for irrigation of complicated agricultural land by using classified airborne digital sensor images. Journal of Indian Society of Remote Sensing, 47, 1307-1314. Retrieved from https://link.springer.com/article/10.1007/s12524-019-01007-4.

Chong, K. L. et al. (2017). A review of remote sensing applications for oil palm studies. Geo-Spatial Information Science, 20(2), 184-200. doi: 10.1080/10095020.2017.1337317.

Clevers, J. G. P. et al. (1994). A framework for monitoring crop growth by combining directional and spectral remote sensing information. Remote Sensing of Environment, 50, 161-170.

Defournya, P. et al. (2019). Near real time agriculture monitoring at national scale at parcel resolution Perfrormance assessment of the sen2 agriculture automated system. Remote Sensing of Environment, $2,551-568$. 
Diepen, K. Van \& Wit, A. De. (2014). The WOFOST model, its principles, implication, main parameters and examples of use, JRC European Commission. Retrieved on November 11, 2019 from https://www.wur.nl/en/Research-Results/Research-Institutes/Environmental-Research/FacilitiesProducts/Software-and-models/WOFOST/Documentation-WOFOST.htm

Du, M. \& Noguchi, N. (2017). Monitoring of wheat growth status and mapping of wheat yield within field spatial variation using color images from UAV Camera system. Remote Sensing, 9(289). doi: $10.3390 /$ rs 9030289.

Ehbrecht, M. et al. (2016). Experimental biodiversity enrichment in oil-palm-dominated landscapes in Indonesia. Frontiers in Plant Science, 07(October), 1-15. doi: 10.3389/fpls.2016.01538.

FAO. (n. d.). GIEWS - Global Information and Early Warning System, Food and Agricultural Organization of the United Nation. Retrieved from http://www.fao.org/giews/background/en/.

Fardusi, M. J., Chianucci, F. \& Barbati, A. (2017). Concept to practice of geospatial-information tools to assist forest management and planning under precision forestry framework: A review. Annals of Silvicultural Research, 41(1), 3-14. doi: 10.12899/asr-1354.

Fieuzal, R., Marais Sicre, C. \& Baup, F. (2016). Estimation of corn yield using multi-temporal optical and radar satellite data and artificial neural networks. International Journal of Applied Earth Observation and Geoinformation, 57, 14-23. doi: 10.1016/j.jag.2016.12.011.

Franch, B. et al. (2019). Remote sensing based yield monitoring application to winter wheat in United States and Ukraine. International Journal Applied Earth Observation Geoinformation, 76, 112-127.

Fritz, S. et al. (2019). A comparison of global agricultural monitoring systems and current gaps. Agricultural Systems, 168(July 2018), 258-272. doi: 10.1016/j.agsy.2018.05.010.

Funk, C. \& Verdin, J. P. (2009). Real-time decision support systems: The femine early warning system network. Spatial Demography, 295-320. doi: https://doi.org/10.1007/978-90-481-2915-7_17.

Geospatial Media and Communication. (2015). Geospatial technology in agriculture industry trends \& prospects 2015 . India.

Ghobadifar, F. et al. (2016). Detection of BPH brown planthopper sheath blight in rice farming using multispectral remote sensing. Geomatics, Natural Hazards and Risk, 7(1), 237-247. doi: 10.1080/19475705.2014.885468.

Grenzdörffer, G. J. (2014). Crop height determination with UAS point clouds. International Archives of the Photogrammetry, Remote Sensing and Spatial Information Sciences - ISPRS Archives, 135-140. doi: 10.5194/isprsarchives-XL-1-135-2014.

Hazir, M. H. M. \& Muda, T. M. T. (2018). The viability of remote sensing for extracting rubber smallholding information: A case study in Malaysia. Egyptian Journal of Remote Sensing and Space Science, (xxxx), 1-13. doi: 10.1016/j.ejrs.2018.05.001. 
Huang, Q. et al. (2011). China crop growth monitoring system-methodology and operational activities overview. International Geoscience and Remote Sensing Symposium (IGARSS), 2961-2964. doi: 10.1109/IGARSS.2011.6049837.

Izzah, A. H. \& Asrina, W. . W. (2019). Black pepper in Malaysia: An overview and future prospects. Agricultural Reviews, 40(4). doi: 10.18805/ag.r-129.

Izzah, A. H., Asrina, W. Y. W. \& Ahmed, O. H. (2017). Spatial variability of NPK in black pepper farm on hilly topography. International conference on Sustainable Soil Management, 103-106.

Kandiannan, K. et al. (2002). Allometric model for leaf area estimation in black pepper. Journal of Agronomy \& Crop Science, $188,138-140$.

Kern, A. et al. (2018). Statistical modelling of crop yield in central Europe using climate data and remote sensing vegetation indices. Agricultural and Forest Meteorology.

Kho, P. E. \& Shang, C. Y. (2016). Novel farming innovation for high production of black pepper (Piper nigrum L.) planting materials. Journal of Agricultural Science and Technology B, 7(5), 23-25. doi: 10.17265/2161-6264/2017.05.001.

Krishnamoorthy, B. \& Parthasarathy, V. A. (2009). Improvement of black pepper. CAB Reviews Perspectives in Agriculture Veterinary Science Nutrition and Natural Resources, 4(85), 1-12. doi: 10.1079/pavsnnr20094085.

Kumar, T. S. S. \& Swarupa, P. U. (2017). Cultivation practices of black pepper in India - A Review. International Journal of Innovative Research in Management Studies, 2(5), 21-27.

Kussul, N. et al. (2018). Crop inventory at regional scale in Ukraine: developing in season and end of season crop maps with multi-temporal optical and SAR satellite imagery. European Journal of Remote Sensing, 51(1), 627-636. doi: 10.1080/22797254.2018.1454265.

Li, H. et al. (2019). Full year crop monitoring and separability assessment with fully polarimetric L-band UAVSAR: Acase study in Sacramento Valley California. International Journal Applied Earth Observation Geoinformation, 1-12. doi: .1037//0033-2909.I26.1.78.

Li, Z. \& Chen, Z. (2011). Remote sensing indicators for crop growth monitoring at different scales. IGARSS. Republic of China: IEEE.

Malaysia Pepper Board. (2018). MPB PenanamanLada.pdf, Panduan Pembajaan Lada Dan Kekurangan Nutrient. Retrieved from mpb.gov.my/mpb/index.php/ms/media4/penerbitan/brosur.

Martinez, A. I. (2017). Precision agriculture for Iraq's and Syria post-conflict recovery. GIS Resources.

Meng, J. H., Wu, B. F. \& Li, Q. Z. (2006). A global crop growth monitoring system based on remote sensing. International Geoscience and Remote Sensing Symposium (IGARSS), (February), 2277-2280. doi: 10.1109/IGARSS.2006.589. 
Ministry of International Trade and Industry. (2018). Industry4WRD: National Policy on Industry 4.0. Malaysia: Ministry of International Trade and Industry.

Nandibewoor, A., Saleem.B.Hebbal \& Hegadid, R. (2015). Remote monitoring of maize through satellite multispectral imagery. In International Conference on Advanced Computing Technologies and Application 2015. India: Procedia Computer Science, 344-353.

Norasma, Y. N. et al. (2018). Rice crop monitoring using multirotor UAV and RGB digital camera at early stage of growth. In IOP Conference Series: Earth Environment Science, 12095. doi: 10.1088/17551315/169/1/012095.

Nurmiaty et al. (2019). Developing agricultural land geospatial information in supporting regional food resilience. In The International Conference on Geoscience.

Orynbaikyzy, A., Gessner, U. \& Conrad, C. (2019). Crop type classification using a combination of optical and radar remote sensing data a review. International Journal of Remote Sensing. doi: 10.1080/01431161.2019.1569791.

Paduit, N. et al. (2018). Nutrient uptake and distribution in black pepper. Better Crops with Plant Food, 102(4), 24-27. doi: $10.24047 / \mathrm{bc} 102424$.

Panda, S. S., Ames, D. P. \& Panigrahi, S. (2010). Application of vegetation indices for agricultural crop yield prediction using neural network techniques. Remote Sensing, 2(3), 673-696. doi: 10.3390/rs2030673.

Prim, A. (2017). An Increasing Role of Geospatial Technologies in Agriculture Management, GIS Resources. Uttar Pradesh, India: Spatial Media and Services Enterprises. Retrieved from www.deimosimaging.com.

Qing, H. et al. (2011). China CGMS methodology and operational activities overview. in IGARSS. China: IEEE.

Raeva, P. L., Šedina, J. \& Dlesk, A. (2019). Monitoring of crop fields using multispectral and thermal imagery from UAV. European Journal of Remote Sensing, 52(sup1), 192-201. doi: 10.1080/22797254.2018.1527661.

Ravindran, P. N. \& Kallupurackal, J. A. (2012). Black pepper. In K.V. Peter (Ed) Handbook of Herbs and Spices (2nd ed.). Woodhead Publishing Limited. doi: 10.1533/9780857095671.86.

Rembold, F. et al. (2017). ASAP - Anomaly hot spots of agriculture production, a new early warning decision support system developed by the Joint Research Centre. In 2017 9th International Workshop on the Analysis of Multitemporal Remote Sensing Images. IEEE. doi: 10.1109/Multi-Temp.2017.8035205.

Rosli, A., Radam, A. \& Rahim, K. A. (2013). Technology adoption in pepper farming: A case study in Sarawak, Malaysia. The Internacional Journal of Social Sciences, 1, 19-22.

Rowshon, M. K. and Amin, M. S. (2010). GIS based irrigation water management for precision farming of rice. International Journal of Agriculture and Biological Engineering, 3(3), 27-35. doi: 10.3965/j.issn.1934-6344.2010.01.027-035. 
Rudiyanto et al. (2019). Automated near-real-time mapping and monitoring of rice extent, cropping patterns, and growth stages in Southeast Asia using Sentinel-1 Time Series on a Google Earth engine platform. Remote Sensing, 11(14), 1666. doi: 10.3390/rs11141666.

Sanny, L., Polla, J. R. \& Fatmawati, I. (2018). The analysis of export potency for Indonesian pepper. Pertanika Journal of Social Sciences \& Humanities, 26, 237-246.

Shafinah, K. et al. (2013). Kebolehcapaian nasihat bagi pengurusan penyakit tanaman oleh pekebun kecil lada hitam, Sarawak : Tinjauan awal. Malaysian Journal of Society and Space, 9(2), 17-26.

Sharifi, M. A. (2000). Crop inventory and production forecasting using remote sensing and agrometeorological models: The case of major agricultural commodities in Hamadan province, Iran. International Archives of Photogrammetry and Remote Sensing, XXXIII, 1364-1372.

Sivaraman, K. et al. (1999). Agronomy of black pepper (Piper nigrum L.) - A review. Journal of Spices and Aromatic Crops, 8(1), 1-18.

Song, Q. et al. (2017). In-season crop mapping with GF-1/WFV data by combining object-based image analysis and random forest. Remote Sensing, 9(11). doi: 10.3390/rs9111184.

Sousa, D. \& Small, C. (2019). Mappring and monitoring rice agriculture with multisensor temporal mixture models.

Srinivasan, V., Srambikkal, H. \& Dinesh, R. (2007). Nutrient management in black pepper. CABI (January).

Su, A. S. M. et al. (2017). Teknologi aplikasi dron untuk pertanian. In Persidangan Kebangsaan Pemindahan Teknologi 2017 (CONFERTECH), (December), 44-48.

Sulok, K. M. T. et al. (2018). Introducing natural farming in black pepper (Piper nigrum L.) cultivation. International Journal of Agronomy, 2018, 1-6. doi: 10.1155/2018/9312537.

Tan, K. P., Kanniah, K. D. \& Cracknell, A. P. (2012). A review of remote sensing based productivity models and their suitability for studying oil palm productivity in tropical regions. Progress in Physical Geography, 36(5), 655-679. doi: 10.1177/0309133312452187.

Wu, B. et al. (2015). Global crop monitoring: A satellite based hierarchical approach. Remote Sensing, 7(4). doi: $10.3390 /$ rs 70403907 .

Xue, J. \& Su, B. (2017). Significant remote sensing vegetation indices: A review of developments and applications. Journal of Sensors, 2017, 1-17. doi: 10.1155/2017/1353691.

Yap, C. A. (2012a). Determination of nutrient uptake characteristic of black pepper (Piper nigrum L.). Journal of Agricultural Science and Technology B, 2, 1091-1099. doi: ISSN 1939-1250.

Yap, C. A. (2012b). Impact of different fertilization methods on the soil, yield and growth performance of black pepper (Piper nigrum L.). Malaysian journal of society and spacesian Journal of Soil Science, 16, 71-87. doi: 1394-7990. 
Yap, C. A. (2016). Efficacy of organic products as black pepper foliar fertilizer. International Journal of Environment, Agriculture and Biotechnology, 1(3), 586-592. doi: 10.22161/ijeab/1.3.42.

Yap, C. A. \& Jarroop, Z. (2018). Residue levels and dissipation behaviors of chlorpyrifos in black pepper berries and soil. Food Research, 2(December), 587-593. doi: https://doi.org/10.26656/fr.2017.2(6).127 Abstract.

Yuzugullu, O. et al. (2017). Determining rice growth stage with xband SAR a metamodel based inversion. Remote Sensing of Environment, 9. doi: 10.3390/rs9050460.

Zainol, W. N. Z. A. et al. (2019). Spectral discrimination of bulloak (Allocasuarina leuhmannii) and associated woodland for habitat mapping of the endangered bulloak jewel butterfly (Hypochrysops piceata) in southern Queensland. Journal of Applied Remote Sensing, 8.

Zambon, I. et al. (2019). Revolution 4.0: Industry vs. agriculture in a future development for SMEs. Processes, 7(1), 36. doi: 10.3390/pr7010036.

Copyright $\odot 2020$ by Kamsan SZL et al., and HH Publisher. This work is licensed under the Creative Commons Attribution-NonCommercial 4.0 International Lisence (CC-BY-NC4.0) 